\section{Protein expression}

AthenaES expression media and cell culture products designed for protein expression studies are now available in North America from Axxora. For researchers involved in recombinant protein expression, ten media optimized for protein expression in Escherichia coli provide up to sevenfold increases in protein expression, says the company. Expression media include standard LB broth, as well as a number of proprietary APF-certified media. Also available are more than a dozen cell culture products, including serum-free formulations for growing human cells, insect cells and for establishing new cell lines.

(+1) 858-550-8830

www.axxora.com

\section{Protein characterization}

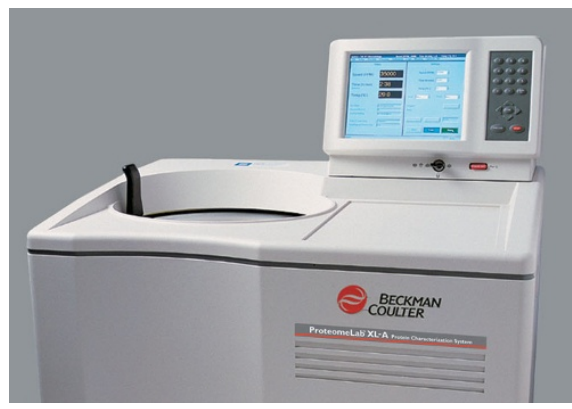

Beckman Coulter's new system for probing the proteome.

Beckman Coulter has introduced the ProteomeLab XL-A,XL-I for protein characterization in solution. Part of the company's new ProteomeLab family of products, the XL-A,XL-I provides measurement of protein heterogeneity, stoichiometry, interacting/self-associating systems and molecular conformation studies for improved lead optimization. The system is suitable for stability testing applications in which drug compound aggregation presents a challenge. In addition to rigorous characterization, the XL-A,XL-I technology measures relative changes in the distribution of molecular weights and enables the collection of many simple yes/no answers, for quick and efficient investigations. The ProteomeLab XL-A,XLI offers a choice of detection systems. The XL-A uses a scanning UV/VIS detection system, whereas the XL-I is equipped with both scanning UV/VIS and Rayleigh inter- ference detection systems. The XL-I can simultaneously collect data from both detection systems to provide the broadest spectrum of data for solution interaction analysis.

Tel. (+1) 800-742-2345/ (+1) 714-871-4848 www.beckmancoulter.com

\section{Improving your image}

This April Bio-Rad launched CellMap - a confocal imaging unit for those on a budget. CellMap is based exclusively on solid-state lasers. Two models are available: CellMapID and CellMapIC. With its $488-\mathrm{nm}$ and $532-\mathrm{nm}$ lasers, CellMapIC is suitable for the visualization of GFP-/FITC/TRITC-/Cy3-labeled proteins and structures. CellMapID, on the other hand, with its 405-nm and 488-nm lasers, is suitable for autofluorescence studies and for studying DAPI-/CFP-stained nuclei and GFP-/FITClabeled proteins in cells. CellMap is initially available on the Nikon TE2000 and E600 microscopes.

Tel. (+44) (0) 20-8328-2000

www.cellscience.bio-rad.com

\section{High-throughput cell- based studies}

BD Biosciences Discovery Labware offers the BD Falcon HTS FluoroBlok 96-multiwell insert system. This cell culture assay platform is composed of a black multiwell insert plate with fluorescence blocking, microporous (3- or 8 - $\mu \mathrm{m}$ pore sizes), lowbinding PET (polyethylene terephthalate) membrane and a 96-well receiver plate and lid. The 96-well insert plate format is compatible with standard fluorescence plate readers, robots and fluid handling instruments. The company says that the wide blocking range of the BD FluoroBlok membrane affords users the flexibility of choosing from a variety of fluorophores for screening compounds in cell-based assays such as chemotaxis, migration and invasion. Each insert is said to have a generous automation-compatible port, making it possible to sample above and below the membrane. The back housing of the 96multiwell insert and receiver plate is designed to minimize crosstalk between the wells.

Tel. (+1) 978-671-1388

www.bdbiosciences.com
IC filters

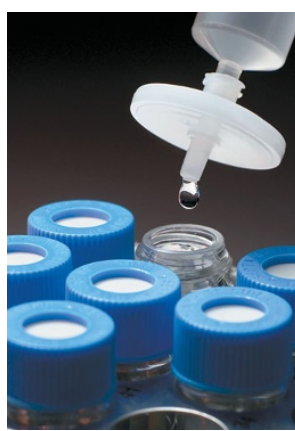

Fine filters for IC work.

Millipore offers the IC Millex Filter Unit, a low-extractable filter for ion chromatography. The filter unit includes a high-density polyethylene housing and low-binding, hydrophilic, polytetrafluroethylene (PTFE) membrane for the removal of fine particulates prior to instrument analysis. To ensure cleaner IC spectras, IC Millex filters are certified for low ion-extractable levels. The membrane is said to clarify both aqueous and mild organic solutions with its $0.2-\mu \mathrm{m}$ pore size. In addition, the IC Millex housing is compatible with commonly used solvents. Individually packaged to minimize the risk of extraneous ionic contamination, IC Millex filter units are available in $13-$ and $25-\mathrm{mm}$ diameters. The 13-mm units are recommended for filtering volumes from 1 to 10 $\mathrm{ml}$; the $25-\mathrm{mm}$ units are recommended for filtering volumes from 10 to $100 \mathrm{ml}$.

Tel. (+1) 800-MILLIPORE

www.millipore.com

\section{Portable incubator}

Breeder is the new soft-sided, portable incubator for use with large-scale multiple deck roller culture systems. It is available from Wheaton Science Products. With a temperature control range of $5^{\circ} \mathrm{C}$ above ambient to $60^{\circ} \mathrm{C}$, it can be used to keep cells warm while preparing a series of cultures for transport to a warm room or incubator. It can also serve as an inexpensive alternative or temporary expansion of a warm room. The Breeder consists of temperature sensors, heaters and fans that enclose the roller rack cell culture in flexible plastic and insulating materials. The company says it is easily placed over a full deck roller culture unit and secured. For convenient storage, the side is collapsed, the unit folded and placed in the compact storage case.

Tel. (+1) 856-825-0400

www.wheatonsci.com/html/index.jsp 